\title{
Relación entre colesterol dietario, consumo de huevo y perfil lipídico en adultos aparentemente sanos, según grupos de edad
}

\author{
Relationship between dietary cholesterol, egg consumption and lipid profile in apparently
} healthy adults according to age group

\author{
Rosa Lorenza Oriondo Gates , Ivonne Bernui Leo1, Lázaro Rubén Valdivieso Izquierdo, \\ Enriqueta Estrada Menacho ${ }^{2}$ \\ 'Centro de Investigación de Bioquímica y Nutrición (CIBN), Facultad de Medicina, Universidad Nacional Mayor de San Marcos, Lima, Perú. \\ 2 Departamento Académico de Nutrición, Facultad Medicina, Universidad Nacional Mayor de San Marcos, Lima, Perú.
}

\begin{abstract}
Resumen
Objetivos: Evaluar la relación entre el colesterol dietario, consumo de huevo y el perfil lipídico en adultos aparentemente sanos, según grupo etario. Diseño: Estudio descriptivo, de asociación cruzada. Institución: Centro de Investigación de Bioquímica y Nutrición, Facultad de Medicina, Universidad Nacional Mayor de San Marcos, Lima, Perú. Participantes: Adultos aparentemente sanos. Intervenciones: En 50 adultos aparentemente sanos se determinó el consumo de colesterol dietario, huevo y perfil lipídico. Resultados: Trece participantes consumian menos de 3 huevos/semana, 19 de 3 a 4 huevos/semana y 18 más de 4 huevos/semana. En relación al consumo de colesterol dietario, el grupo de 20 a 29 años presentó un promedio de ingesta $(358,9 \pm 152,5 \mathrm{mg} / \mathrm{dL})$ significativamente superior $(p<0,05)$ al grupo de 30 a 39 años $(223 \pm 82,8 \mathrm{mg} / \mathrm{dL}$ ). El colesterol (col) total de los tres grupos según consumo de huevo fue $179,8 \pm 52,168,8 \pm 44$ y $164,3 \pm 62 \mathrm{mg} / \mathrm{dL}$, respectivamente; para el HDL-col se obtuvo $38,9 \pm 15,28,0 \pm 15$ y $35,2 \pm 14 \mathrm{mg} / \mathrm{dL}$, respectivamente; para el LDL-col, 118,1 $\pm 41,112,5 \pm 39$ y 100,4 $\pm 47,9 \mathrm{mg} / \mathrm{dL}$, respectivamente; para los triglicéridos, 199,2 \pm 169, $152,8 \pm 108$ y 124,2 $\pm 92 \mathrm{mg} / \mathrm{dL}$, respectivamente. Conclusiones: El consumo de colesterol dietario y huevo en la muestra estudiada no tuvo relación con el perfil lipídico.

Palabras clave: Huevos, lípidos, colesterol en la dieta.

Abstract

Objectives: To determine the relationship between dietary cholesterol, egg consumption and lipid profile in apparently healthy adults according to age group. Design: Descriptive cross-association study. Setting: Biochemistry and Nutrition Research Center, Faculty of Medicine, Universidad Nacional Mayor de San Marcos. Participants: Apparently healthy adults. Interventions: In 50 apparently healthy adults dietary cholesterol consumption, egg and lipid profile were determined. Results: Thirteen participants consumed less than 3 eggs / week, 19 consumed from 3-4 eggs / week and 18 consumed more than 4 eggs / week. The 20-29 year-old group had an average intake of dietary cholesterol $(358,9 \pm 152,5 \mathrm{mg} / \mathrm{dL})$ significantly higher $(p<0,05)$ than the group 30-39 year-0ld $(223 \pm 82,8 \mathrm{mg} /$ $\mathrm{dL}$ ). Total cholesterol (chol) of the three groups according to egg consumption was respectively $179,8 \pm 52,168,8 \pm 44$, and 164,3 \pm $62 \mathrm{mg} / \mathrm{dL}$; for HDL-chol 38,9 $\pm 15,28,0 \pm 15$, and 35,2 $\pm 14 \mathrm{mg} / \mathrm{dL}$; for LDL-chol 118,1 $\pm 41,112,5 \pm 39$, and 100,4 $\pm 47,9 \mathrm{mg} / \mathrm{dL}$; for triglycerides 199, $2 \pm 169,152,8 \pm 108,124,2 \pm 92 \mathrm{mg} / \mathrm{dL}$. Conclusions: The consumption of dietary cholesterol and eggs in the sample was not related to the lipid profile.
\end{abstract}

Key words: Eggs, lipids, dietary cholesterol.

An Fac med. 2013;74(1):27-30

\section{INTRODUCCIÓN}

La historia señala que la esperanza de vida ha ido en aumento, pero las causas de muerte han ido cambiando. En 1900, la primera causa de muerte era la neumonía y las enfermedades cardiovasculares estaban en cuarto lugar; en 1950, las enfermedades cardiovasculares pasaron al primer lugar como causa de muerte, manteniéndose así hasta el año $2000^{(1)}$.

Desde 1940, se comenzó las mediciones de colesterol plasmático en pacientes y aquellos que lo tenían alto según las estadísticas de la época, se les aconsejaba disminuir en la dieta el consumo de colesterol, particularmente el consumo de huevos de gallina ${ }^{(2)}$.

En los últimos 40 años, el público ha sido aconsejado en contra del consumo de huevo por la preocupación acerca del riesgo de padecer de enfermedad coronaria, basado en que el huevo es una fuente rica en colesterol dietario ${ }^{(3,4)}$.

La contribución del colesterol dietario al riesgo de las enfermedades cardiovasculares permanece controversial.
Aún más, la literatura epidemiológica no demuestra que el consumo de huevo sea un factor de riesgo para la enfermedad coronaria ${ }^{(5)}$.

Según la Encuesta Nacional de Indicadores Nutricionales, Bioquímicos, Socio-económicos y Culturales Relacionados con las Enfermedades Crónicas Degenerativas, realizado por el Centro Nacional de Alimentación y Nutrición ${ }^{(6)}$, se encontró que la prevalencia de hipercolesterolemia se elevaba de $6 \%$ en el grupo de 20 a 30 años hasta $33,6 \%$ en el grupo mayor de 60 
años. Así mismo, se ha encontrado una prevalencia de síndrome metabólico de $16,8 \%$, en base a la información de esa misma encuesta ${ }^{(7)}$. Teniendo en consideración la recomendación de reducir el consumo de huevo a tres veces por semana, es que nos preocupa que esta restricción podría ser para quienes tengan problemas de hipercolesterolemia, y no sea aplicable al resto de la población.

El huevo como alimento es económico y bajo en calorías, fuente de nutrientes tales como folato, riboflavina, selenio, colina y vitaminas $B_{12}$ y $A$. También, es una de las pocas fuentes exógenas de vitaminas $\mathrm{K}$ y $\mathrm{D}$. Así mismo, proporciona proteínas de alto valor biológico ${ }^{(8,9)}$. Aunque el huevo es rico en colesterol, tiene grasas insaturadas que favorecen la salud cardíaca y vascular ${ }^{(10)}$. Estos beneficios nutricionales del huevo son para las diferentes etapas de la vida y a un precio menor que otras fuentes igualmente nutritivas. La matriz lipídica de la yema sirve para mejorar la biodisponibilidad de carotenoides, como la luteína y la zeaxantina, que previenen la degeneración macular relacionada con la edad (pérdida de visión que ocurre con el transcurso de los años) e intervienen disminuyendo la incidencia de cataratas ${ }^{(11,12)}$.

La información obtenida de individuos de vida libre muestra que el consumo de huevos no está asociado con niveles más altos de colesterol. Song y Kerver ${ }^{(13)}$ examinaron la asociación entre el consumo de huevo y el colesterol sérico en más de 20000 participantes, en la 3 a Encuesta Nacional de Salud y Nutrición de los Estados Unidos (NHANES III), y hallaron que quienes consumieron un huevo o menos a la semana tuvieron un promedio de colesterol ligeramente alto en comparación con aquellos que consumieron 4 o más huevos por semana (197 versus $193 \mathrm{mg} /$ $\mathrm{dL}, \mathrm{p}<0,01)$. Un estudio que examinó la ingesta de alimentos en 117000 enfermeras y profesionales de la salud, durante un periodo de 14 años, no encontró diferencia en el riesgo relativo para la enfermedad cardiovascular entre aquellos que consumían menos de un huevo a la semana y los que consumían más de un huevo al día ${ }^{(14)}$.

Conocer si el consumo de huevo no afecta el nivel del perfil lipídico, ayudaría a flexibilizar las recomendaciones respecto al consumo de huevo, alimento altamente nutritivo y económico en las poblaciones que más lo necesitan.

El objetivo del presente trabajo fue evaluar la relación entre el colesterol dietario, consumo de huevo y el perfíl lipídico (colesterol total, HDL-colesterol, LDL-colesterol y triglicéridos), en adultos aparentemente sanos y según grupo etario.

\section{MÉTODOS}

El presente fue un estudio descriptivo de asociación cruzada. La población estuvo constituida por adultos de 20 a 59 años, de uno y otro sexo. El tamaño de muestra fue de 50 adultos aparentemente sanos, libre de enfermedades conocidas, capaces de responder la encuesta de consumo y que no tomaban medicamentos.

El método de selección de la muestra fue de tipo no probabilístico.

La convocatoria fue a través de avisos dentro de la Facultad de Medicina. A los participantes se les explicó acerca del estudio y se absolvieron las dudas que hubieron. Luego, se les citó para una fecha posterior en la que se les indicó que acudieran en ayunas (de 8 a 12 horas). Previa firma de consentimiento informado, se procedió a la toma de muestra de sangre. Luego, se hizo la encuesta de frecuencia de consumo semicuantitativa de alimentos, para poder determinar el consumo de huevo y aporte de colesterol.

Se separó el suero de las muestras de sangre obtenidas, y se analizó los parámetros bioquímicos colesterol total, LDL-colesterol, HDL-colesterol y triglicéridos, mediante el método enzimático (kit Valtek Lab), en los laboratorios del CIBN ${ }^{(15)}$.
Los valores de referencia de las pruebas bioquímicas fueron: colesterol total $<200 \mathrm{mg} / \mathrm{dL}, \mathrm{HDL}$-colesterol (hombres) $>35 \mathrm{mg} / \mathrm{dL}$, HDL-colesterol (mujeres) $>45 \mathrm{mg} / \mathrm{dL}$; LDL-colesterol $<130 \mathrm{mg} / \mathrm{dL}$; triglicéridos $<150 \mathrm{mg} /$ dL, según el Manual de diagnóstico clínico de Valtek ${ }^{(15)}$.

Los datos, después de su revisión y limpieza, fueron digitados en una base en el programa MS EXCEL. Se formó tres grupos de personas, según la cantidad de huevos consumidos: menos de 3 por semana, de 3 a 4 por semana y más de 4 por semana.

Al aplicar la prueba de KolmogorovSmirnov, se encontró que solo el colesterol total y la fracción LDL seguían una distribución normal, mientras que la fracción HDL, los triglicéridos y el número de huevos consumidos por semana no seguían dicha distribución. Para la estadistica descriptiva, se calculó los valores promedio y desviación de las variables numéricas por cada grupo etario. Para conocer la relación entre el consumo de huevos y el perfil lipídico, se comparó el promedio de colesterol total, HDL-col y LDL-col y triglicéridos entre los tres grupos formados, según consumo de huevos, con la prueba Kruskall-Wallis, con un nivel de confianza de $95 \%$. Las pruebas fueron realizadas en el programa SPSS versión 15.

\section{RESULTADOS}

La muestra estuvo constituida por 50 personas, 29 hombres y 21 mujeres, con edad promedio de 40,6 \pm 14 años.

La muestra fue separada en dos grupos de edad y se comparó el número de huevos consumidos por semana, encontrándose que el grupo de 20 a 29 años $(n=16)$ tuvo un consumo promedio de 5,1 $\pm 3,0$ huevos/ semana y el de 30 a 59 años $(n=34)$, un consumo promedio de 3,5 $\pm 2,1$ huevo/semana. Aunque se observó la tendencia a consumir más huevos entre los más jóvenes, esta diferencia no fue significativa $(p=0,051)$. 
Tabla 1. Distribución de los participantes según grupo de edad y consumo de huevos por semana.

\begin{tabular}{|c|c|c|c|c|c|c|c|c|}
\hline \multirow{2}{*}{$\begin{array}{l}\text { Grupo de edad } \\
\text { (años) }\end{array}$} & \multicolumn{2}{|c|}{ Menos de 3 huevos } & \multicolumn{2}{|c|}{ De 3 a 4 huevos } & \multicolumn{2}{|c|}{ Más de 4 huevos } & \multicolumn{2}{|c|}{ Total } \\
\hline & $n$ & $\%$ & $\mathrm{n}$ & $\%$ & $n$ & $\%$ & $\mathrm{n}$ & $\%$ \\
\hline 20 a 29 & 1 & 6,3 & 7 & 43,8 & 8 & 50,0 & 16 & 100 \\
\hline 30 a 59 & 12 & 35,3 & 12 & 35,3 & 10 & 29,4 & 34 & 100 \\
\hline Total & 13 & 26,0 & 19 & 38,0 & 18 & 36,0 & 50 & 100 \\
\hline
\end{tabular}

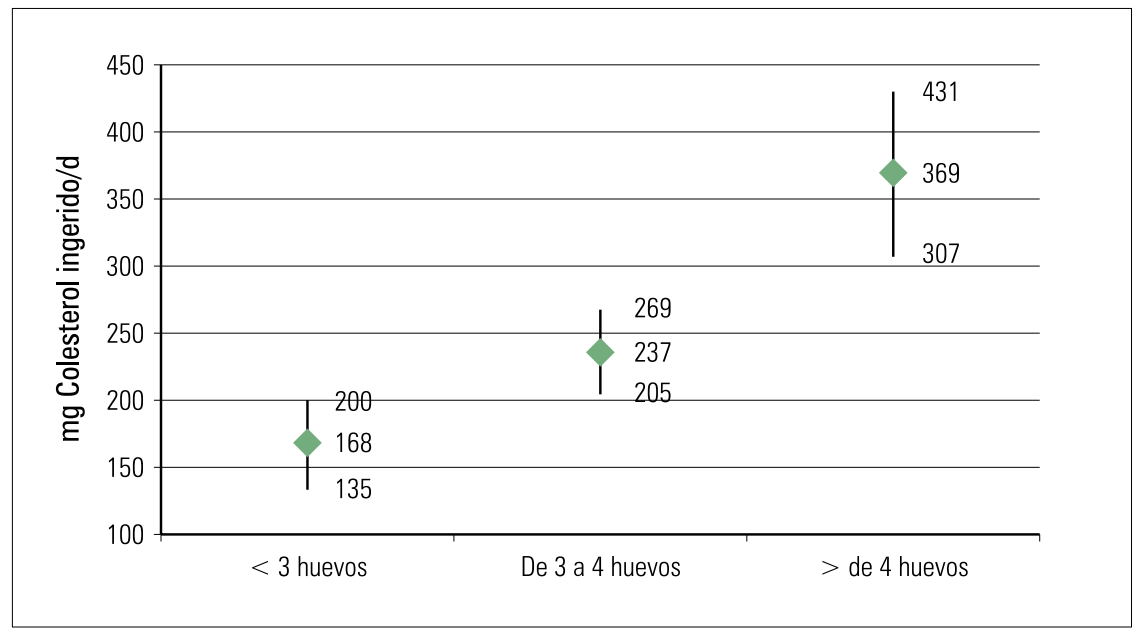

Figura 1. Promedio e intervalo de confianza del colesterol dietario (mg), según consumo de huevo/ semana.

La mitad de las personas entre 20 y 29 años consumía más de 4 huevos/ semana (tabla 1), mientras que las personas de 30 a 59 años estaban distribuidas de manera proporcional en los tres grupos. No obstante, esta diferencia de proporciones del consumo de huevo según grupo de edad no fue significativa $(\mathrm{p}>0,05)$.

El consumo de colesterol dietario de las personas de 20 a 29 años tuvo un promedio de ingesta $(358,9 \pm 152,5 \mathrm{mg} /$ $\mathrm{dL})$ significativamente mayor $(\mathrm{p}<0,05)$ que las personas entre 30 a 39 años $(223 \pm 82,8 \mathrm{mg} / \mathrm{dL})$.
En cuanto al consumo de huevo y colesterol dietario, se halló que aquellos que consumieron más de 4 huevos por semana presentaron (gráfica 1) una mayor ingesta de colesterol en comparación a los que consumieron menos de tres huevos por semana, con diferencia significativa $(\mathrm{p}<0,05)$.

Los promedios de colesterol total, HDL-colesterol, LDL-colesterol y triglicéridos, según grupos de edad, fueron mayores para las personas de mayor edad (tabla 2) y esta diferencia fue significativa en todos los casos $(p<0,05)$.
Así mismo, se comparó el promedio de los lípidos en sangre, según el consumo semanal de huevo. Llama la atención que el promedio de los triglicéridos en las personas que consumían menos de 3 huevos/semana estaba por encima de lo normal $(199,2 \mathrm{mg} / \mathrm{dL})$. No se encontró diferencia significativa entre los grupos ( $p>0,05$; tabla 3 ).

Se encontró valores por encima de los límites 'deseados' de colesterol total en 13 personas, HDL disminuidos en 27 personas, LDL aumentado en 16 personas y triglicéridos incrementados en 20 de los encuestados. Así mismo, se halló un mayor número de personas en el grupo de mayor edad (30 a 59 años) con niveles de perfil lipídico por encima de los límites deseables ${ }^{(15,16)}$.

\section{DISCUSIÓN}

El promedio de colesterol dietario fue mayor en el grupo de menor edad y sorprende que dicho promedio $(358,9$ $\mathrm{mg} / \mathrm{día}$ ) fuera mayor a lo recomendado por la American Heart Association -Sociedad Americana de Cardiología (300 $\mathrm{mg} / \mathrm{d})^{(16)}$.

También, se encontró que aquellos que consumían más huevo, tenían una mayor ingesta de colesterol; pero, esta mayor ingesta no se vio reflejada en un perfil lipídico elevado. Keys y Parlin ${ }^{(17)}$ calcularon que el consumo de un huevo diario solo aumenta el colesterol en sangre en $4 \%$ en sujetos normocolesterolémicos. De manera semejante, en diferentes estudios se informa que el consumo de huevo no está relacionado con un mayor nivel de colesterol ${ }^{(13)}$; tampoco está relacionado con la aparición de la enfermedad cardiovascular ${ }^{(14)}$.

Tabla 2. Promedio y desviación estándar del colesterol total, HDL-colesterol, LDL-colesterol y triglicéridos, según grupo de edad.

\begin{tabular}{|c|c|c|c|c|c|c|c|c|c|}
\hline \multirow{2}{*}{$\begin{array}{l}\text { Grupo de edad } \\
\text { (años) }\end{array}$} & \multirow[t]{2}{*}{$\mathrm{n}$} & \multicolumn{2}{|c|}{$\begin{array}{l}\text { Colesterol total } \\
(\mathrm{mg} / \mathrm{dL})\end{array}$} & \multicolumn{2}{|c|}{$\begin{array}{l}\text { HDL-col } \\
(\mathrm{mg} / \mathrm{dL})\end{array}$} & \multicolumn{2}{|c|}{$\begin{array}{l}\text { LDL-col } \\
(\mathrm{mg} / \mathrm{dL})\end{array}$} & \multicolumn{2}{|c|}{$\begin{array}{l}\text { Triglicéridos } \\
\text { (mg/dL) }\end{array}$} \\
\hline & & Media & $\mathrm{DE}$ & Media & $\mathrm{DE}$ & Media & $\mathrm{DE}$ & Media & $\mathrm{DE}$ \\
\hline 20 a 29 & 16 & 135,6 & 48,8 & 24,7 & 14,6 & 86,9 & 46,1 & 135,6 & 48,8 \\
\hline 30 a 59 & 34 & 185,9 & 48,6 & 37,5 & 14,1 & 120,3 & 37,1 & 185,9 & 48,6 \\
\hline
\end{tabular}


Tabla 3. Promedio y desviación estándar del HDL-colesterol, LDL-colesterol y triglicéridos por grupos, según consumo semanal de huevos.

\begin{tabular}{|c|c|c|c|c|c|c|c|c|c|}
\hline \multirow{2}{*}{$\begin{array}{c}\text { Huevos / } \\
\text { semana }\end{array}$} & \multirow[t]{2}{*}{$\mathrm{n}$} & \multicolumn{2}{|c|}{$\begin{array}{l}\text { Col-total } \\
\text { (mg/dL) }\end{array}$} & \multicolumn{2}{|c|}{$\begin{array}{l}\text { HDL-col } \\
\text { (mg/dL) }\end{array}$} & \multicolumn{2}{|c|}{$\begin{array}{l}\text { LDL-col } \\
(\mathrm{mg} / \mathrm{dL}) \\
\end{array}$} & \multicolumn{2}{|c|}{$\begin{array}{c}\text { Triglicéridos } \\
\text { (mg/dL) }\end{array}$} \\
\hline & & Media & Desv. típ. & Media & Desv. típ. & Media & Desv. típ. & Media & Desv. típ. \\
\hline Menos de 3 & 13 & 179,8 & 51,5 & 38,9 & 15,4 & 118,1 & 41,1 & 199,2 & 169,8 \\
\hline De 3 a 4 & 19 & 168,8 & 44,3 & 28,0 & 15,3 & 112,5 & 39,1 & 152,8 & 108,1 \\
\hline Más de 4 & 18 & 164,3 & 61,7 & 35,2 & 14,4 & 100,4 & 47,9 & 124,2 & 92,0 \\
\hline
\end{tabular}

La aparición de las enfermedades cardiovasculares involucra otros factores modificables, como lo son la actividad física, consumo de tabaco, consumo de alcohol, entre otros ${ }^{(14)}$.

Se ha observado la tendencia de un mayor consumo de huevos entre los más jóvenes, probablemente porque había varios deportistas que lo consumían, tanto la clara como la yema.

Se encontró que $26 \%$ de los sujetos encuestados tenía el colesterol sérico elevado. Este hallazgo es ligeramente mayor a la prevalencia de hipercolesterolemia $(18,5 \%)$ en sujetos de 20 a 60 años encontrada por la Encuesta Nacional de Indicadores Nutricionales y Bioquímicos relacionados con enfermedades crónicas degenerativas (ENIN) ${ }^{(6)}$. Así mismo se halló en el presente estudio un aumento de los lípidos sanguíneos con la edad; tendencia similar ha sido encontrada en la encuesta nacional antes mencionada ${ }^{(6)}$.

Los huevos hacen contribuciones a las ingestas dietarias de muchos otros nutrientes, incluyendo proteína, grasa monoinsaturada, ácido linoleico, retinol, vitamina $D, \alpha$-tocoferol, folato, vitamina $\mathrm{B}_{2}$, vitamina $\mathrm{B}_{12}$, que por sí solos podrían tener un efecto protector sobre las enfermedades cardiovasculares.

Entre las limitaciones del presente estudio debe mencionarse el hecho de no haber podido contar con una muestra aleatoria y que por lo tanto nuestros hallazgos son válidos solamente para la muestra estudiada.

Se concluye que el consumo de colesterol dietario y huevo en la muestra estudiada no tuvo relación con el perfil lipídico.

\section{REFERENCIAS BIBLIOGRÁFICAS}

1. Kritchevsky S. A review of scientific research and recommendations regarding eggs. J Am Coll Nutr. 2004;23(6):596S-600S

2. Valenzuela A, Morgado N. Breve historia de la relación entre el colesterol y las enfermedades cardiovasculares. Rev chil nutr. [revista en la Internet]. 2006 Ago [citado 2012 Mayo 30]; 33(2): 130-134. Disponible en: http://www.scielo. $\mathrm{cl} /$ scielo.php?script=sci_arttext\&pid=S071775182006000200001 \&lng=es.

3. Page IH, Allen NB, Chamberlain FL, Keys A, Stamler $J$, Stare FJ. Dietary fat and its relation to heart attacks and stroke. Circulation. 1961;23:133-6.

4. American Heart Association. Diet and Coronary Heart Disease. Dallas: American Heart Association. 1973-

5. McNamara DJ. The impact of egg limitations on coronary heart disease risk: do the numbers add up? J Am Coll Nutr. 2000;19:540S-548S.

6. Ministerio de Salud. Instituto Nacional de Salud. CENAN. Encuesta Nacional de Indicadores Nutricionales, Bioquímicos, Socioeconómicos y Culturales Relacionados con las Enfermedades Crónicas Degenerativas. Lima, Perú. 2006.

7. Pajuelo J, Sánchez J. El síndrome metabólico en adultos, en el Perú. An Fac med. 2007;68(1):3846.

8. USDA National Nutrient Database for Standard Reference. 2012. Disponible en : http://www.ars. usda.gov/Services/docs.htm?docid $=22113$

9. Ministerio de Salud. Instituto de Nutrición. Tabla

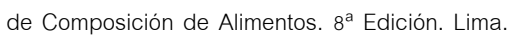
2008.

10. Qureshi Al, Suri FK, Ahmed S, Nasar A, Divani AA, Kirmani JF. Regular egg consumption does not increase the risk of stroke and cardiovascular diseases. Med Sci Monit. 2007;13:CR1-8.
11. Ribaya-Mercado JD, Blumberg JB. Lutein and zeaxanthin and their potential roles in disease prevention. J Am Coll Nutr. 2004;23(90006):567S$587 \mathrm{~S}$.

12. Greene C, Waters D, Clark R, Contois J, Fernandez ML. Plasma LDL and HDL characteristics and carotenoid content are positively influenced by egg consumption in an elderly population. Nutrit Metab. 2006;3:6.

13. Song WO, Kerver, JM. Nutritional contribution of eggs to American diets. J Am Coll Nutr. 2000;19(5):556S-562S.

14. Hu FB, Stampfer MJ, Rimm EB, Manson JE, Ascherio A, Colditz GA, Rosner BA, Spiegelman D, Speizer FE, Sacks FM, Hennekens CH, Willett WC. A prospective study of egg consumption and risk of cardiovascular disease in men and women. JAMA. 1999;281:1387-94.

15. Valtek. Manual de técnicas para diagnóstico clínico. Santiago de Chile: Nuñoa. 2000.

16. Krauss RM, Eckel RH, Howard B, Appel LJ, Daniels SR, Deckelbaum RJ, et al. AHA Dietary Guidelines. Revision 2000: A statement for healthcare professionals from the nutrition committee of the American Heart Association. Stroke. 2000;31(11):2751-66.

17. Keys A, Parlin RW. Serum-cholesterol response to changes in dietary lipids. Am J Clin Nutr. 1966;19:175-81.

Trabajo presentado el 8 de agosto de 2012 y aceptado para publicación el 13 de setiembre de 2012.

Fuente de financiamiento:

Recursos propios y del CIBN

Conflicto de Interés: Ninguno

Correspondencia:

Rosa Lorenza Oriondo Gates

Dirección: Urb. Taboadita Mz. B1, 12 Bellavista, Callao

Teléfono: 420 8918. Celular: 999756214

Correo electrónico: rosa.oriondo@gmail.com 